\title{
No touch radiofrequency ablation for hepatocellular carcinoma: a conceptual approach rather than an iron law
}

\author{
Olivier Seror ${ }^{1,2,3}$ \\ ${ }^{1}$ Unité de Radiologie Interventionnelle, Hôpital Avicenne, Hôpitaux Universitaires Paris-Seine-Saint-Denis, Assistance Publique Hôpitaux de Paris, \\ Bobigny, France; ${ }^{2}$ Unité Mixte de Recherche 1162, Génomique Fonctionnelle des Tumeurs Solides, Institut National de la Santé et de la Recherche \\ Médicale, Paris 7, Paris, France; ${ }^{3}$ Unité de Formation et de Recherche Santé Médecine et Biologie Humaine, Université Paris 13, Communauté \\ d'Universités et Etablissements Sorbonne Paris Cité, Bobigny, France \\ Correspondence to: Olivier Seror. MD, PhD. Unité Fonctionnelle de Radiologie Interventionnelle, Hôpital Avicenne, Hôpitaux Universitaires Paris- \\ Seine-Saint-Denis, 125 rue de Stalingrad, 93000 Bobigny, France. Email: olivier.seror@aphp.fr. \\ Comment on: Lee DH, Lee MW, Kim PN, et al. Outcome of No-Touch Radiofrequency Ablation for Small Hepatocellular Carcinoma: A Multicenter \\ Clinical Trial. Radiology 2021;301:229-36.
}

Submitted Nov 30, 2021. Accepted for publication Dec 10, 2021.

doi: $10.21037 / \mathrm{hbsn}-21-512$

View this article at: https://dx.doi.org/10.21037/hbsn-21-512

Using conventional intra-tumoral radiofrequency ablation (IT-RFA) for the treatment of hepatocellular carcinoma (HCC) $3 \mathrm{~cm}$ or smaller, high local tumor recurrence rates have been reported (up to $20 \%$ ) despite initial complete ablation rates reaching $95 \%$. This prompted the recommendation of no touch RFA (NT-RFA) for this indication in clinical practice since 2006 (1). The rationale of NT-RFA is based on the assumption that the insertion of electrodes no longer inside but deliberately just outside the borders of the tumors could allow achieving more consistent tumoral ablation with safety margins, hence preventing further secondary local progression from unablated extranodular peripheral microscopic tumoral foci (2). Obviously, full compliance with no-touch insertions of electrodes rules out the risk of tumor seeding which has been reported as high as $12 \%$ (3). However, the worldwide cumulative experience of IT-RFA for the treatment of HCC had previously shown that the incidence of seeding is in fact less than $1 \%$, thus far below the secondary local tumor progression due to the lack of sufficient ablation margin (4). Another potential advantage of strict compliance with NTRFA over IT-RFA is to ablate the tumor with less intratumoral pressure with a subsequent diminished risk of per procedural metastasis due to intravascular release of tumor cells (5). Of course, tangible evidence of this third advantage of NT-RFA is difficult to demonstrate $(6,7)$.

Since its introduction, NT-RFA has been the matter of numerous clinical studies (8). Three among them, including one randomized trial comparing NT-RFA with IT-RFA for the treatment of HCC, confirmed the superiority of no touch approach in the prevention of local or subsegmental recurrence, however without evident translation in terms of overall survival (Table 1) (7-9). Pursuing their considerable scientific efforts, Lee et al had recently published a multicenter clinical trial aimed at evaluating midterm clinical outcomes of NT-RFA of small HCC smaller $2.5 \mathrm{~cm}$ (10). Successful no touch procedures without violation of the tumor were achieved in 128 of the 140 (91.4\%) patients enrolled. The cumulative 2-years local recurrence rate was as low as $1.6 \%$. In five centers, six operators with different skills in imaging-guided liver tumor ablation ranging from 5 to 25 years were able to reproduce excellent performances of NT-RFA in term of sustained complete response. However, it is interesting to point out that these results were obtained in patients selected on more stringent criteria than in the previous multicentric study of Hocquelet et al. (8). In the latter, although larger HCCs up to $5 \mathrm{~cm}$ and whatever their proximity to the capsule or large vessels within the liver were ablated using NT-RFA, the 2-years local recurrence rate $(7 \%)$ was still advantageously very low when compared to those reported following IT-RFA procedures (8). Such results strongly suggest that NT-RFA could improve outcomes of a much wider spectrum of HCC patients than those enrolled in Lee et al. study (10). As the size of the margin recommended to achieve is directly correlated to the size of the ablated tumor, the application of a no touch 
Table 1 Comparative studies of no-touch versus intra tumorous radiofrequency ablation for the treatment of HCC

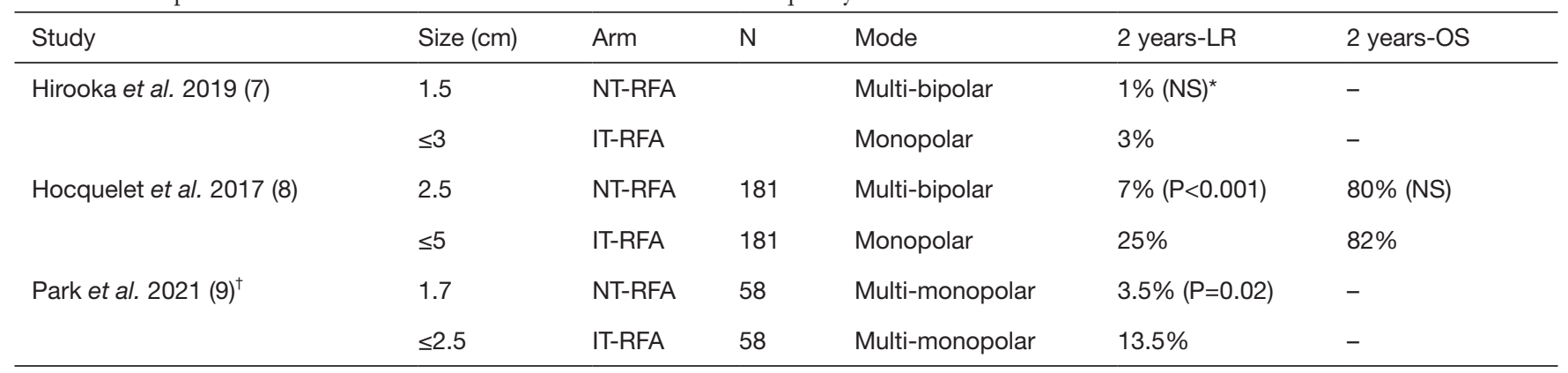

*, Statistically non-significant after inverse probability of treatment weighting adjustment but intrasubsegmental recurrence defined as intrasegmental recurrence $>5 \mathrm{~mm}$ distant from border of the tumor, was still significantly lower in NT-RFA group (1\% versus $18 \%$ at 2 -year). ${ }^{\dagger}$, Randomized trial. HCC, hepatocellular carcinoma; NT, no touch; IT, intratumorous; RFA, radiofrequency ablation; NS, non-significant; LR, local recurrence; OS, overall survival.

approach is in this context preferable (11). In that respect, the recommendation of a $5 \mathrm{~mm}$ margin for guided HCC ablative therapies must be regarded as a minimal goal since an improved recurrence-free survival has been reported in patients resected with at least a $2 \mathrm{~cm}$ versus $1 \mathrm{~cm}$ margin (11). Moreover, larger is the tumor higher is the probability that it abuts the liver capsule or a large vessel. Thus, in our routine practice of NT-RFA more than $30 \%$, $10 \%$ and $20 \%$ patients have HCC larger than $3 \mathrm{~cm}$ and/or in subcapsular location and/or abutting large vessel, respectively.

Some technological points are key considerations for clinical application of NT-RFA technique and acceptance of no touch concept for ablation in liver. The technique was first described using a multiprobe RF ablative device able to feed sequentially in bipolar mode up to six separate linear electrodes that is up to 15 combinations of dipoles, each in their own orientations (1). Thus, this technological approach was named multi-bipolar mode to hight the difference with multi-monopolar mode of most other monopolar ablative devices able to also feed several probes but independently. In comparison with multi-monopolar, multi-bipolar mode allows much more energy deposition inside than outside the arrangement of electrodes and therefore to perform the ablation from the periphery to the center of this arrangement (centripetal ablation) and not to every direction from each applicator (centrifugal ablation) (Figure 1) (12). Because the predictability of tumor-free margin is the main stake of no touch concept, the centripetal ablation allowed by multi-bipolar technology appears clearly more suitable than centrifugal ablation of the multi-monopolar devices.

The multi-bipolar technology enables not only no touch ablation of HCC larger than $3 \mathrm{~cm}$ but also in other various often coexisting challenging situations such as subcapsular (or even exophytic) or perivascular locations using declinations of no touch electrodes insertion strategies as retro-nodular convergent and cutting ablations (13). Moreover, the wide range of geometrical modulations of no touch energy deposition allowed by multi-bipolar technology enables the ablation of tumors poorly delineated as subsegmental infiltrative forms often associated with intra-hepatic portal invasion (14). For similar reasons, multi-bipolar NT-RFA is a relevant method to accommodate the marginal error of coregistration of fusion imaging guidance recommended for the ablation of small inconspicuous liver targets with ultrasound (Figure 1). Using common centrifugal ablative technologies, a limited mismatch between real time ultrasonography and referral imaging 3D set (MRI or CT) is associated with higher risk of treatment failure than with centripetal multibipolar NT-RFA.

Finally, since the first clinical use of NT RFA for the treatment of HCC, sharing their own experiences with the techniques other searchers have shown that this approach can clearly improve the outcome of patients within current indications of ablation $(7,9,10)$. The time has come to regard the NT-RFA beyond its capacity to ablate small HCC without resort to intra tumorous puncture, as a major opportunity to extend the spectrum of patients amenable to ablation. First for BCLC 0 and A patients for whom ablation using common intratumorous techniques is deemed to be infeasible because of unfavourable locations or too poor visibility at real time imaging. Second for patients bearing tumor larger than $3 \mathrm{~cm}$, even ill-limited and infiltrative by applying variant strategies of electrodes derived from former no touch technique. In other words, NT-RFA should be now considered not an iron law of 


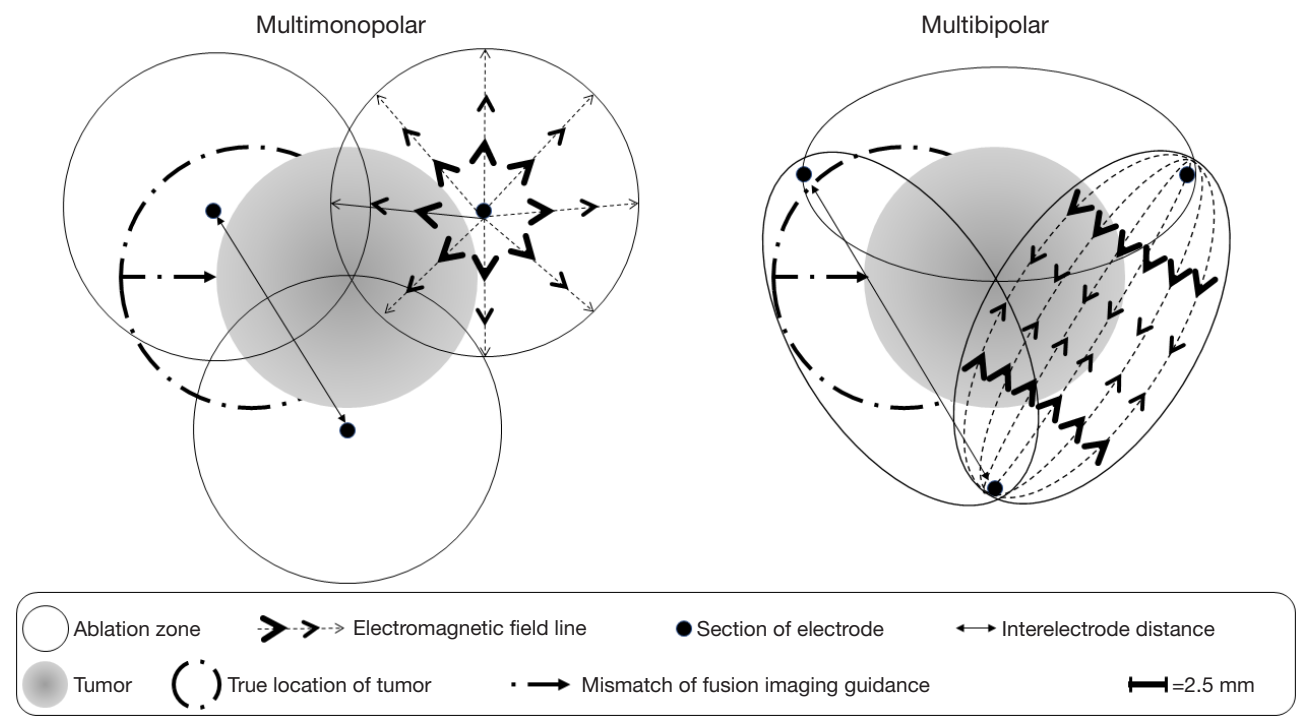

Figure 1 Bull's eye diagrams of no-touch radiofrequency ablation of tumor using 3 straight electrodes in multi-monopolar versus multibipolar mode. In multi-monopolar mode each electrode induces in perpendicular axis a circular centrifugal radiating electromagnetic typically enables an ablation of $2 \mathrm{~cm}$ maximal diameter around the electrode. The ablation of the entire tumor and the margin requires the overlaps of each ablation zones that depends on interelectrode distance (with the current monopolar devices available in clinical practice, $1.7 \mathrm{~cm}$ is the maximal interelectrode distance recommended to achieve consistently continuous ablation between two electrodes). In multibipolar mode each pair of electrodes induces in perpendicular axis elliptical centripetal radiating electromagnetic typically enables an ablation of $2.5 \mathrm{~cm} \times 1.5 \mathrm{~cm}$ between the electrodes. To ensure the coverage of the entire tumor and the margin requires the overlaps of each elliptical ablation zones induced by each pair of electrodes that the long axis is strongly linked to interelectrode distance (with the current bipolar devices available in clinical practice, $3 \mathrm{~cm}$ is the maximal interelectrode distance recommended to achieve consistently continuous ablation between two electrodes). Thus, this diagram shows clearly that using 3 separate straight electrodes for the no-touch ablation of a same $1.7 \mathrm{~cm}$ diameter tumor, comparing with the multi-monopolar mode the multi-bipolar mode allows easier coverage of the tumor including a more consistent thick of ablative margin. Moreover, the multi-bipolar mode that allows longer interelectrode distance insertions accommodates better than multi-monopolar mode a possible mistargeting due to unperfect coregistration of real-time ultrasonography with referral volume imaging set (CT or MRI) when insertions of electrodes are performed under fusion imaging guidance. On this diagram a $6 \mathrm{~mm}$ mismatch between the two imaging modalities leads to incomplete ablation with multi-monopolar mode while with multi-bipolar mode the entire tumor is still covered.

extra tumoral technique but a wider conceptual approach of centrifugal ablation useable in various challenging situations. We strongly believe that the emergence of this new paradigm for ablation of HCC is closely linked to our cumulative experience of RFA based on the use of multibipolar technology (15).

\section{Acknowledgments}

Funding: None.

\section{Footnote}

Provenance and Peer Review: This article was commissioned by the editorial office, Hepatobiliary Surgery and Nutrition. The article did not undergo external peer review.

Conflicts of Interest: The author has completed the ICMJE uniform disclosure form (available at https://hbsn. amegroups.com/article/view/10.21037/hbsn-21-512/coif). The author has no conflicts of interest to declare.

Ethical Statement: The author is accountable for all aspects of the work in ensuring that questions related to the accuracy or integrity of any part of the work are appropriately investigated and resolved.

Open Access Statement: This is an Open Access article 
distributed in accordance with the Creative Commons Attribution-NonCommercial-NoDerivs 4.0 International License (CC BY-NC-ND 4.0), which permits the noncommercial replication and distribution of the article with the strict proviso that no changes or edits are made and the original work is properly cited (including links to both the formal publication through the relevant DOI and the license). See: https://creativecommons.org/licenses/by-nc-nd/4.0/.

\section{References}

1. Seror O, N'Kontchou G, Nault JC, et al. Hepatocellular Carcinoma within Milan Criteria: No-Touch Multibipolar Radiofrequency Ablation for Treatment-Long-term Results. Radiology 2016;280:611-21.

2. Seror O, N'Kontchou G, Van Nhieu JT, et al. Histopathologic comparison of monopolar versus no-touch multipolar radiofrequency ablation to treat hepatocellular carcinoma within Milan criteria. J Vasc Interv Radiol 2014;25:599-607.

3. Llovet JM, Vilana R, Brú C, et al. Increased risk of tumor seeding after percutaneous radiofrequency ablation for single hepatocellular carcinoma. Hepatology 2001;33:1124-9.

4. Livraghi T, Lazzaroni S, Meloni F, et al. Risk of tumour seeding after percutaneous radiofrequency ablation for hepatocellular carcinoma. Br J Surg 2005;92:856-8.

5. Tanaka T, Yamanaka N, Oriyama T, et al. Factors regulating tumor pressure in hepatocellular carcinoma and implications for tumor spread. Hepatology 1997;26:283-7.

6. Kotoh K, Nakamuta M, Morizono S, et al. A multistep, incremental expansion method for radio frequency ablation: optimization of the procedure to prevent increases in intra-tumor pressure and to reduce the ablation time. Liver Int 2005;25:542-7.

7. Hirooka M, Hiraoka A, Ochi H, et al. Prospective cohort trial to confirm the efficacy of no-touch radio frequency ablation. J Gastroenterol Hepatol 2019;34:567-74.

8. Hocquelet A, Aubé C, Rode A, et al. Comparison of notouch multi-bipolar vs. monopolar radiofrequency ablation for small HCC. J Hepatol 2017;66:67-74.

9. Park SJ, Cho EJ, Lee JH, et al. Switching Monopolar NoTouch Radiofrequency Ablation Using Octopus Electrodes for Small Hepatocellular Carcinoma: A Randomized Clinical Trial. Liver Cancer 2021;10:72-81.

10. Lee DH, Lee MW, Kim PN, et al. Outcome of NoTouch Radiofrequency Ablation for Small Hepatocellular Carcinoma: A Multicenter Clinical Trial. Radiology 2021;301:229-36.

11. Shi M, Guo RP, Lin XJ, et al. Partial hepatectomy with wide versus narrow resection margin for solitary hepatocellular carcinoma: a prospective randomized trial. Ann Surg 2007;245:36-43.

12. Seror O. Ablative therapies: Advantages and disadvantages of radiofrequency, cryotherapy, microwave and electroporation methods, or how to choose the right method for an individual patient? Diagn Interv Imaging 2015;96:617-24.

13. Petit A, Hocquelet A, N'kontchou G, et al. No-Touch Multi-bipolar Radiofrequency Ablation for the Treatment of Subcapsular Hepatocellular Carcinoma $\leq 5 \mathrm{~cm}$ Not Puncturable via the Non-tumorous Liver Parenchyma. Cardiovasc Intervent Radiol 2020;43:273-83.

14. N'Kontchou G, Nault JC, Sutter O, et al. Multibipolar Radiofrequency Ablation for the Treatment of MassForming and Infiltrative Hepatocellular Carcinomas > 5 cm: Long-Term Results. Liver Cancer 2019;8:172-85.

15. Seror O, Hocquelet A, Sutter O. Could Monopolar Mode be a Suitable Strategy of Energy Deposition for Performing No-Touch Radiofrequency Ablation of Liver Tumor $\leq 5 \mathrm{~cm}$ ? Cardiovasc Intervent Radiol 2018;41:1630-1.
Cite this article as: Seror O. No touch radiofrequency ablation for hepatocellular carcinoma: a conceptual approach rather than an iron law. HepatoBiliary Surg Nutr 2022;11(1):132-135. doi: $10.21037 / \mathrm{hbsn}-21-512$ 